\title{
Thieno [3,2-d] pyrimidin-4-one Derivatives as Potential Antibacterial Agents
}

\author{
Chander Mohan ${ }^{1}$, Gaurav Bhargava ${ }^{1}$ and Preet M.S. Bedi ${ }^{*}$ \\ ${ }^{1}$ Department of Applied Chemistry, ${ }^{2}$ Department of Pharmaceutical Sciences, \\ Guru Nanak Dev University, Amritsar 143 005, Punjab, India
}

KEYWORDS Pyrimidinones. Antibacterial Agent. Thio-Claisen. Gram Positive Bacteria. Gram Negative Bacteria

\begin{abstract}
Several thieno $[3,2-d]$ pyrimidin-4-ones (4a-i) were conveniently prepared by the thio-claisen rearrangement of 5-prop-2-ynylsulfanyl pyrimidin-4-ones derivatives. The constitution of the products was supported by IR, PMR and mass spectral study. The compounds synthesized were tested in vitro against gram positive microorganisms, Bacillus subtilis, Bacillus cereus, Staphlococus aureus, Enterococcus faecalis and gram negative microorganism Eseherichia coli, Pseudomonas aeruginosa, Proteus vulgaris, Klabsiella pneumoniae and Shigella sonnei. Standard drugs were also tested under identical conditions for comparing the results.
\end{abstract}

\section{INTRODUCTION}

The emergence and spread of bacterial resistance represent a severe global problem (Ehlert 1999). Multidrug-resistance gram-positive pathogens, such as methicillin-resistant, Staphylococci aureus (MRSA), penicillinresistant Streptococcus pneumoniae (PRSP), and vancomycin-resistant enterrococci (VRE), have become a serious problem in the medical community (Cunha 1998; Dalhoff 1994) One particular alarming sign is the acquisition of resistance to vancomycin, an antibiotic generally regarded as the agent of last resort for serious infections (File 1999 ; Witte 1999; Endtz et al. 1999). In the field of quinoline antibacterial agents, various attempts have been made to obtain potent drugs for resistant gram-positive bacteria such as torvafloxin (Gootz et al. 1992), moxifloxacin (Petersen et al.1996), gemifloxacin (Hong et al. 1997) and gatifloxacin (Hirai et al. 1990) etc, have been developed and introduced into clinical use over the past several years. Considering that the incidence of gram-positive bacterial resistance to antibacterial agents has been growing, however, antibacterial activities of these newer quinolines are not potent enough and bacteria resistant to

"For Correspondance:

Dr. Preet M.S.Bedi, Reader,

Department of Pharmaceutical Sciences,

Guru Nanak Dev University,

Amritsar-143 005, Punjab, India.

Telephone: +91-183-2258802-09 Extension: 3459

Fax: +91-183-2258819-20

E-mail: bedi_preet@yahoo.com those agents will be problematic in the near future (Ho et al. 2000; Weiss et al. 2001). Therefore, there is an urgent need for novel chemical entities that are particularly effective against gram-positive pathogens including the multiresistant strains. In the last few years, various pyrimidinone derivatives exhibited a wide range of activity such as antitumor, antiviral, antitubercular and antifungal activities (Maria et al. 2004). In continuation of our efforts in developing heterocycles of biological interest (Bedi et al. 2004), we wish to report here the antimicrobial activities of the title thieno[3,2-d]pyrimidinones derivatives in order to study their antibacterial activities.

\section{MATERIALS AND METHODS}

Melting points were determined by open capillary using Veego Precision Digital Melting Point apparatus (MP-D) and are uncorrected. IR spectra were recorded on a Shimadzu D-8001 spectrophotometer. ${ }^{1} \mathrm{H}$ NMR spectra were recorded in deuterochloroform with Bruker AC-E 200 (200 MHz) spectrometers using TMS as internal standard. Chemical shift values are expressed as ppm downfield from TMS and J values in $\mathrm{Hz}$. Splitting patterns are indicated as $\mathrm{s}$ : singlet, $\mathrm{d}$ : doublet, t: triplet, $\mathrm{m}$ : multiplet, q: quartet, br: broad peak and brs: broad singlet. ${ }^{13} \mathrm{C}$ NMR spectra were also recorded on Bruker AC-200E (50.4 MHz) spectrometers in deuterochloroform using TMS as internal standard. Mass spectra were recorded on Shimadzu GCMS-QP-2000 mass spectrometer. 
Elemental analyses were performed on Heraus Carbon, Hydrogen, Nitrogen-Oxygen Rapid Elemental Analyzer. Column chromatography was performed on a silica gel (60-120) mesh or Harrison Research Chromatotron using $2 \mathrm{~mm}$ plates (Silica gel 60 PF254).

\section{Synthesis}

The required thieno $[3,2-d]$ pyrimidin-4-ones (4a-i) were conveniently prepared by the thioclaisen rearrangement of 5-prop-2-ynylsulfanyl pyrimidin-4-ones derivatives 3 . The pyrimidinone derivatives 3 were in turn obtained by the [4+2] cycloaddition reaction of 1,3-diazabuta-1,3-dienes $\mathbf{1}$ with prop-2-ynylsulfanyl ketene $\mathbf{2}$, generated in situ from the corresponding acid and $p$-toluene sulfonyl chloride in the presence of triethylamine in dry methylene chloride at room temperature. Thermolysis of pyrimidinones $\mathbf{3}$ in refluxing chlorobenzene, resulted in the formation of thieno $[3,2-d]$-pyrimdinones (4a-i) as crystalline solids in good yields $(75-80 \%)$ (Scheme 1). The thieno [3,2-d] pyrimidinones so obtained were characterized on the basis of analytical and spectral evidences mentioned in the experimental section.

Physical and spectral data of representative compounds are as follows.

6-Methyl-2-phenyl-3-o-tolyl-3H-thieno[3,2d]pyrimidin-4-one (4a).creamish solid, yield 77\% ; m.p. 237-238 $\mathrm{C}$; Anal. Calcd for $\mathrm{C}_{20} \mathrm{H}_{16} \mathrm{~N}_{2} \mathrm{OS}$ : C, 72.26; H, 4.85; N, 8.43. Found: C, 72.35; H, 4.91; $\mathrm{N}, 8.28 \%: v_{\text {max }}(\mathrm{KBr}): 1685 \mathrm{~cm}^{-1} ; \mathrm{d}_{\mathrm{H}}(200 \mathrm{MHz}$, $\left.\mathrm{CDCl}_{3}\right): 2.34\left(\mathrm{~s}, 3 \mathrm{H}, \mathrm{CH}_{3}\right) ; 2.69\left(\mathrm{~s}, 3 \mathrm{H}, \mathrm{CH}_{3}\right) ; 7.23-$ $7.52\left(\mathrm{~m}, 10 \mathrm{H}, \mathrm{ArH}\right.$ and $\left.\mathrm{H}_{\mathrm{d}}\right) ; \mathrm{d}_{\mathrm{C}}\left(50.4 \mathrm{MHz}, \mathrm{CDCl}_{3}\right)$ : $16.2\left(\mathrm{CH}_{3}\right), 19.2\left(\mathrm{CH}_{3}\right), 114.0,120.7,122.8,123.8$, 127.9, 129.2, 129.9, 130.3, 134.7, 149.8, 155.7, 156.8, 157.9, and 159.5; m/z $332\left(\mathrm{M}^{+}\right)$.

6-Methyl-2-methylsulfanyl-3-phenyl-3Hthieno[3,2-d]pyrimidin-4-one (4b). creamish solid, yield $82 \%$; m.p. $218-219^{\circ} \mathrm{C}$; Anal. Calcd for $\mathrm{C}_{14} \mathrm{H}_{12} \mathrm{~N}_{2} \mathrm{OS}_{2}$ : C, 58.31; H, 4.19; N, 9.71. Found: C, 58.47; H, 4.11; N, 9.63\%: $v_{\text {max }}(\mathrm{KBr}): 1676 \mathrm{~cm}^{-1}$; $\mathrm{d}_{\mathrm{H}}\left(200 \mathrm{MHz}, \mathrm{CDCl}_{3}\right): 2.70\left(\mathrm{~s}, 3 \mathrm{H}, \mathrm{CH}_{3}\right) ; 2.89(\mathrm{~s}$, $\left.3 \mathrm{H}, \mathrm{SCH}_{3}\right) ; 7.23-7.42\left(\mathrm{~m}, 6 \mathrm{H}, \mathrm{ArH}\right.$ and $\left.\mathrm{H}_{\mathrm{d}}\right) ; \mathrm{d}_{\mathrm{C}}$ $\left(50.4 \mathrm{MHz}, \mathrm{CDCl}_{3}\right): 16.4\left(\mathrm{SCH}_{3}\right), 16.8\left(\mathrm{CH}_{3}\right), 116.2$, $129.5,123.8,127.5,128.8,149.3,155.1,157.7,158.2$ and $159.0 ; \mathrm{m} / \mathrm{z} 288\left(\mathrm{M}^{+}\right)$.

6-Methyl-2-methylsulfanyl-3-p-tolyl-3Hthieno[3,2-d]pyrimidin-4-one (4c). white solid, yield $75 \%$; m.p. $212-213^{\circ} \mathrm{C}$; Anal. Calcd for $\mathrm{C}_{15} \mathrm{H}_{14} \mathrm{~N}_{2} \mathrm{OS}_{2}: \mathrm{C}, 59.57 ; \mathrm{H}, 4.67 ; \mathrm{N}, 9.26$. Found: C,
59.82; H, 4.58; N, 9.10\%: $v_{\text {max }}(\mathrm{KBr}): 1670 \mathrm{~cm}^{-1} ; \mathrm{d}_{\mathrm{H}}$ $\left(200 \mathrm{MHz}, \mathrm{CDCl}_{3}\right): 2.34\left(\mathrm{~s}, 3 \mathrm{H}, \mathrm{CH}_{3}\right) ; 2.67(\mathrm{~s}, 3 \mathrm{H}$, $\left.\mathrm{CH}_{3}\right) ; 2.90$ (s, 3H, $\left.\mathrm{SCH}_{3}\right) ; 6.98-7.12$ (m, 5H, $\mathrm{ArH}$ and $\left.\mathrm{H}_{\mathrm{d}}\right) ; \mathrm{d}_{\mathrm{C}}\left(50.4 \mathrm{MHz}_{2} \mathrm{CDCl}_{3}\right): 16.5\left(\mathrm{SCH}_{3}\right), 16.7$ $\left(\mathrm{CH}_{3}\right), 19.0\left(\mathrm{CH}_{3}\right), 115.3,119.7,123.5,127.9,128.9$, 148.5, 154.5, 157.2, 158.1 and 159.3; m/z $302\left(\mathrm{M}^{+}\right)$.

3- (4-Methoxyphenyl)-6-methyl-2-methyl sulfanyl-3H-thieno[3,2-d]pyrimidin-4-one (4d). white solid, yield $88 \%$; m.p. $215-216^{\circ} \mathrm{C}$; Anal. Calcd for $\mathrm{C}_{15} \mathrm{H}_{14} \mathrm{~N}_{2} \mathrm{O}_{2} \mathrm{~S}_{2}$ : C, 56.58; H, 4.43; N, 8.80. Found: C, 56.49; H, 4.68; N, 8.64\%: $v_{\text {max }}(\mathrm{KBr})$ : $1668 \mathrm{~cm}^{-1} ; \mathrm{d}_{\mathrm{H}}\left(200 \mathrm{MHz}, \mathrm{CDCl}_{3}\right): 2.68(\mathrm{~s}, 3 \mathrm{H}$, $\left.\mathrm{CH}_{3}\right) ; 2.90\left(\mathrm{~s}, 3 \mathrm{H}, \mathrm{SCH}_{3}\right) ; 3.75\left(\mathrm{~s}, 3 \mathrm{H}, \mathrm{OCH}_{3}\right) ; 7.05-$ 7.27 (m, 5H, ArH and $\left.\mathrm{H}_{\mathrm{d}}\right) ; \mathrm{d}_{\mathrm{C}}\left(50.4 \mathrm{MHz}, \mathrm{CDCl}_{3}\right)$ : $16.4\left(\mathrm{SCH}_{3}\right), 16.7\left(-\mathrm{CH}_{3}\right), 55.7\left(\mathrm{OCH}_{3}\right), 114.7,120.5$, $123.5,127.9,130.1,150.2,156.4,157.1,157.9$ and $159.0 ; \mathrm{m} / \mathrm{z} 318\left(\mathrm{M}^{+}\right)$.

3- (4-Chlorophenyl)-6-methyl-2-methyl sulfanyl-3H-thieno[3,2-d]pyrimidin-4-one (4e). pale yellow solid, yield: $78 \%$; m.p. $202-203^{\circ} \mathrm{C}$; Anal. Calcd for $\mathrm{C}_{14} \mathrm{H}_{1} \mathrm{ClN}_{2} \mathrm{OS}_{2}$ : C, 51.70; H, 4.03; $\mathrm{N}, 8.62$. Found: C, $51.89 ; \mathrm{H}, 3.95 ; \mathrm{N}, 8.58 \%$ : $v_{\text {, }}$ (KBr): $1668 \mathrm{~cm}^{-1} ; \mathrm{d}_{\mathrm{H}}\left(200 \mathrm{MHz}, \mathrm{CDCl}_{3}\right): 2.36(\mathrm{~s}$, $\left.3 \mathrm{H}, \mathrm{CH}_{3}\right) ; 2.92\left(\mathrm{~s}, 3 \mathrm{H}, \mathrm{SCH}_{3}\right) ; 6.98-7.12(\mathrm{~m}, 5 \mathrm{H}$, $\mathrm{ArH}$ and $\left.\mathrm{H}_{\mathrm{d}}\right) ; \mathrm{d}_{\mathrm{C}}\left(50.4 \mathrm{MHz}, \mathrm{CDCl}_{3}\right): 16.6\left(\mathrm{SCH}_{3}\right)$, $16.7\left(\mathrm{CH}_{3}\right), 115.6,119.7,123.8,127.7,128.8,148.7$, 154.5, 157.4, 158.3 and 159.5; m/z $290\left(\mathrm{M}^{+}\right)$.

2,3-Diphenyl-6-methyl-3H-thieno[3,2d]pyrimidin-4-one (4f).creamish solid, yield 78\% ; m.p. 223-224 ${ }^{\circ} \mathrm{C}$; Anal. Calcd for $\mathrm{C}_{19} \mathrm{H}_{14} \mathrm{~N}_{2} \mathrm{OS}$ : C, 71.67; H, 4.43; N, 8.80. Found: C, 71.81; H, 4.27; $\mathrm{N}, 8.82 \% \cdot v_{\max }(\mathrm{KBr}): 1665 \mathrm{~cm}^{-1} ; \mathrm{d}_{\mathrm{H}}(200 \mathrm{MHz}$, $\left.\mathrm{CDCl}_{3}\right): 2.70\left(\mathrm{~s}, 3 \mathrm{H}, \mathrm{CH}_{3}\right) ; 7.15-7.31(\mathrm{~m}, 11 \mathrm{H}, \mathrm{ArH}$ and $\left.\mathrm{H}_{\mathrm{d}}\right) ; \mathrm{d}_{\mathrm{C}}\left(50.4 \mathrm{MHz}, \mathrm{CDCl}_{3}\right): 17.2\left(\mathrm{CH}_{3}\right), 116.2$, 118.9, 122.3, 126.0, 128.8, 129.2, 129.9, 134.7, 149.6, $155.2,156.8,157.5$ and $158.8 ; \mathrm{m} / \mathrm{z} 318\left(\mathrm{M}^{+}\right)$.

6-Methyl-2-phenyl-3-p-tolyl-3H-thieno[3,2d]pyrimidin-4-one (4g).light yellow solid, yield $78 \%$; m.p. $231-233^{\circ} \mathrm{C}$; Anal. Calcd for $\mathrm{C}_{20} \mathrm{H}_{16} \mathrm{~N}_{2} \mathrm{OS}$ : C, 72.26; H, 4.85; N, 8.43. Found: C, 72.18; H, 4.99; N, 8.41\%: $v_{\max }(\mathrm{KBr}): 1670 \mathrm{~cm}^{-1}$; $\mathrm{d}_{\mathrm{H}}\left(200 \mathrm{MHz}, \mathrm{CDCl}_{3}\right): 2.28\left(\mathrm{~s}, 3 \mathrm{H}, \mathrm{CH}_{3}\right) ; 2.63(\mathrm{~s}$, $\left.3 \mathrm{H}, \mathrm{CH}_{3}\right) ; 7.00(\mathrm{~d}, 2 \mathrm{H}, \mathrm{J}=8.4 \mathrm{~Hz}, \mathrm{ArH}) ; 7.08(\mathrm{~d}, 2 \mathrm{H}$, $\mathrm{J}=8.4 \mathrm{~Hz}, \mathrm{ArH}) ; 7.18-7.30\left(\mathrm{~m}, 6 \mathrm{H}, \mathrm{ArH}\right.$ and $\left.\mathrm{H}_{\mathrm{d}}\right) ; \mathrm{d}_{\mathrm{C}}$ (50.4 MHz, $\left.\mathrm{CDCl}_{3}\right)$ : $16.7\left(\mathrm{CH}_{3}\right), 19.6\left(\mathrm{CH}_{3}\right), 114.0$, 120.2, 122.8, 127.5, 128.5, 129.0, 129.7, 130.3, 136.0, $150.1,155.7,157.1,157.7$, and 159.0; m/z $332\left(\mathrm{M}^{+}\right)$.

3- (4-Methoxyphenyl)-6-methyl-2-phenyl$3 \mathrm{H}$-thieno[3,2-d]pyrimidin-4-one (4h). white solid, yield 75\%; m.p. $220-221^{\circ} \mathrm{C}$; Anal. Calcd for $\mathrm{C}_{20} \mathrm{H}_{16} \mathrm{~N}_{2} \mathrm{O}_{2} \mathrm{~S}$ : C, 68.94; H, 4.63; N, 8.04. Found: C, 68.73; H, 4.73; N, 8.19\%: $v_{\max }(\mathrm{KBr}) ; 1680 \mathrm{~cm}^{-1} ; \ddot{\mathrm{a}}_{\mathrm{H}}$ (200MHz): 2.64 (s, 3H, $\left.\mathrm{CH}_{3}\right) ; 3.74\left(\mathrm{~s}, 3 \mathrm{H}, \mathrm{OCH}_{3}\right)$; 
$6.76(\mathrm{~d}, 2 \mathrm{H}, \mathrm{J}=8.8 \mathrm{~Hz}, \operatorname{ArH}) ; 6.99(\mathrm{~d}, 2 \mathrm{H}, \mathrm{J}=8.8 \mathrm{~Hz}$, $\mathrm{ArH})$; 7.05-7.33 (m, 6H, ArH and $\left.\mathrm{H}_{\mathrm{d}}\right) ; \mathrm{d}_{\mathrm{C}}(50.4$ $\left.\mathrm{MHz}, \mathrm{CDCl}_{3}\right)$ : $16.7\left(\mathrm{CH}_{3}\right) ; 55.2\left(\mathrm{OCH}_{3}\right) ; 114.1$, 120.8, 123.5, 127.9, 128.9, 129.0, 129.9, 130.1, 135.5, 150.2, 156.6, 157.0, 157.8, 159.1: $m / z 348\left(\mathbf{M}^{+}\right)$.

3-(4-Chlorophenyl)-6-methyl-2-phenyl-3Hthieno[3,2-d]pyrimidin-4-one (4i).creamish solid, yield $80 \%$; m.p. $239-240^{\circ} \mathrm{C}$; Anal. Calcd for $\mathrm{C}_{19} \mathrm{H}_{13} \mathrm{ClN}_{2} \mathrm{OS}$ : C, 64.68; $\mathrm{H}, 3.71 ; \mathrm{N}, 7.94$. Found: $\mathrm{C}, 64.97 ; \mathrm{H}, 3.58 ; \mathrm{N}, 7.78 \%$ : $v_{\text {max }}(\mathrm{KBr}): 1680 \mathrm{~cm}^{-}$ ${ }^{1} ; \mathrm{d}_{\mathrm{H}}\left(200 \mathrm{MHz}, \mathrm{CDCl}_{3}\right): 2.65\left(\mathrm{~s}, 3 \mathrm{H}, \mathrm{CH}_{3}\right) ; 6.96$ (d, $2 \mathrm{H}, \mathrm{J}=8.4 \mathrm{~Hz}, \mathrm{ArH}) ; 7.04$ (d, 2H, J=8.4 Hz, ArH); 7.18-7.30 (m, 6H, ArH and $\left.\mathrm{H}_{\mathrm{d}}\right)$; $\mathrm{d}_{\mathrm{C}}(50.4 \mathrm{MHz}$, $\left.\mathrm{CDCl}_{3}\right)$ : $16.7\left(\mathrm{CH}_{3}\right), 115.1,121.0,123.7,127.9,128.6$, $129.2,129.8,130.3,135.7,150.0,156.8,157.2,158.1$ and $159.5 ; \mathrm{m} / \mathrm{z} 352\left(\mathrm{M}^{+}\right)$.

\section{Antibacterial Activity}

The antibacterial activity of the test compounds was determined by agar well diffusion method (Aparna et al. 2005) against gram positive microorganisms, Bacillus subtilis MTCC 121, Bacillus cereus MTCC 1272, Staphlococus aureus MTCC 1430, Enterococcus faecalis MTCC 439 and gram negative microorganism Eseherichia coli MTCC 42, Pseudomonas aeruginosa MTCC 1034, Proteus vulgaris MTCC 744, Klabsiella pneumoniae MTCC 109 and Shigella sonnei MTCC 2957 at $1 \mathrm{mg} / \mathrm{ml}$ concentration, using dimethyl sulfoxide (DMSO) as solvent. The bacteria were subcultured on Mueller Hinton Agar Medium. Standard antibacterial ciprofloxacin was also screened under similar conditions for comparison. The Petri dishes were incubated at $37^{\circ} \mathrm{C}$ for $48 \mathrm{~h}$ to know the bacterial growth inhibition developed around the hole and was measured in millimeter for particular test solution with particular organism.

\section{RESULTS AND DISCUSSION}

It has been observed that the compounds (4a4i) when tested for the different strains of gram positive and gram negative bacteria exhibited interesting antibacterial activity, however with a degree of variation. Results as shown in Table 1,2 and Fig 1,2 revealed that compound $\mathbf{4 c}$ and $\mathbf{4 d}$ showed significant antibacterial activity whereas compounds $\mathbf{4 f - 4 i}$ showed decrease in antibacterial activity against gram positive and gram negative strains as compared to the ciprofloxacin taken as standard.. However, compound $\mathbf{4 f}$ did not show any activity against

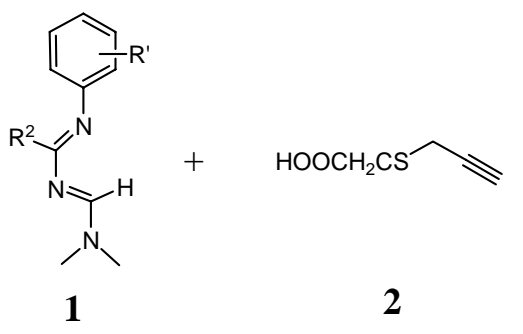

$4 \mathrm{a} \mathrm{R}^{\prime}=\mathrm{o}-\mathrm{CH}_{3} ; \mathrm{R}^{2}=\mathrm{Ph}$

$4 b R^{\prime}=H ; R^{2}=S M e$

$4 c \mathrm{R}^{\prime}=p-\mathrm{CH}_{3} ; \mathrm{R}^{2}=\mathrm{SMe}$

$4 d R^{\prime}=p-\mathrm{OCH}_{3} ; \mathrm{R}^{2}=\mathrm{SMe}$

4 e $\mathrm{R}^{\prime}=p-\mathrm{Cl} ; \mathrm{R}^{2}=\mathrm{SMe}$
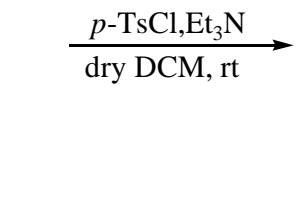

$4 \mathrm{f} \mathrm{R}^{\prime}=\mathrm{H} ; \mathrm{R}^{2}=\mathrm{Ph}$

$4 \mathrm{~g} \mathrm{R}^{\prime}=p-\mathrm{CH}_{3} ; \mathrm{R}^{2}=\mathrm{Ph}$

$4 \mathrm{~h} \mathrm{R}=p-\mathrm{OCH}_{3} ; \mathrm{R}^{2}=\mathrm{Ph}$

$4 \mathrm{i} \mathrm{R}^{\prime}=p-\mathrm{Cl} ; \mathrm{R}^{2}=\mathrm{Ph}$

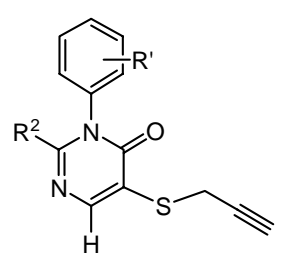

3

refluxing chlorobenzene

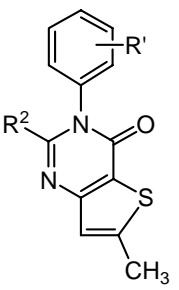

4

Scheme 1. Synthesis of Thieno [3,2,d] pyrimidin-4-one derivatives 
Table 1: In vitro antibacterial activity of compounds 4a-i (zone of inhibition in $\mathrm{mm}^{\mathrm{a}}$ ) against gram positive bacteria

\begin{tabular}{lcccc}
\hline Compound & $\begin{array}{c}\text { B. } \\
\text { subtilis }\end{array}$ & $\begin{array}{c}B . \\
\text { cereus }\end{array}$ & $\begin{array}{c}\text { S. } \\
\text { aureus }\end{array}$ & $\begin{array}{c}\text { E. } \\
\text { faecalis }\end{array}$ \\
\hline $4 \mathrm{a}$ & ++ & + & ++ & + \\
$4 \mathrm{~b}$ & + & ++ & +++ & ++ \\
$4 \mathrm{c}$ & +++ & ++ & + & +++ \\
$4 \mathrm{~d}$ & ++ & +++ & + & ++ \\
$4 \mathrm{e}$ & ++ & + & + & ++ \\
$4 \mathrm{f}$ & - & + & + & + \\
$4 \mathrm{~g}$ & + & + & ++ & + \\
$4 \mathrm{~h}$ & + & + & + & - \\
$4 \mathrm{i}$ & + & + & + & + \\
Cipro & +++ & ++ & +++ & ++ \\
DMSO & - & - & - & - \\
\hline a $(11-15 \mathrm{~mm})$, & ++ & $(16-20 \mathrm{~mm})$, & +++ & $(21-25 \mathrm{~mm})$ and - \\
(inactive). & & & &
\end{tabular}

Table 2: In vitro antibacterial activity of compounds 4a-i (zone of inhibition in $\mathrm{mm}^{\mathrm{a}}$ ) against gram negative bacteria

\begin{tabular}{|c|c|c|c|c|c|}
\hline Compound & $\begin{array}{c}P . \\
\text { aerugi- } \\
\text { nosa }\end{array}$ & $\begin{array}{c}P . \\
\text { vulgaris }\end{array}$ & $\begin{array}{c}\text { K. } \\
\text { pneu- } \\
\text { moniae }\end{array}$ & $\begin{array}{c}S . \\
\text { sonnei }\end{array}$ & $\begin{array}{r}E . \\
\text { coli }\end{array}$ \\
\hline $4 a$ & ++ & ++ & + & ++ & ++ \\
\hline $4 b$ & + & ++ & + & + & ++ \\
\hline $4 c$ & +++ & ++ & ++ & +++ & ++ \\
\hline $4 d$ & + & ++ & + & ++ & +++ \\
\hline $4 \mathrm{e}$ & + & + & ++ & + & + \\
\hline $4 \mathrm{f}$ & + & + & + & ++ & + \\
\hline $4 \mathrm{~g}$ & + & + & + & + & + \\
\hline $4 \mathrm{~h}$ & + & - & + & + & + \\
\hline $4 \mathrm{i}$ & + & + & + & + & + \\
\hline Cipro & ++ & ++ & ++ & +++ & +++ \\
\hline DMSO & - & - & - & - & - \\
\hline
\end{tabular}

$\mathrm{a}+(11-15 \mathrm{~mm}),++(16-20 \mathrm{~mm}),+++(21-25 \mathrm{~mm})$ and (inactive).

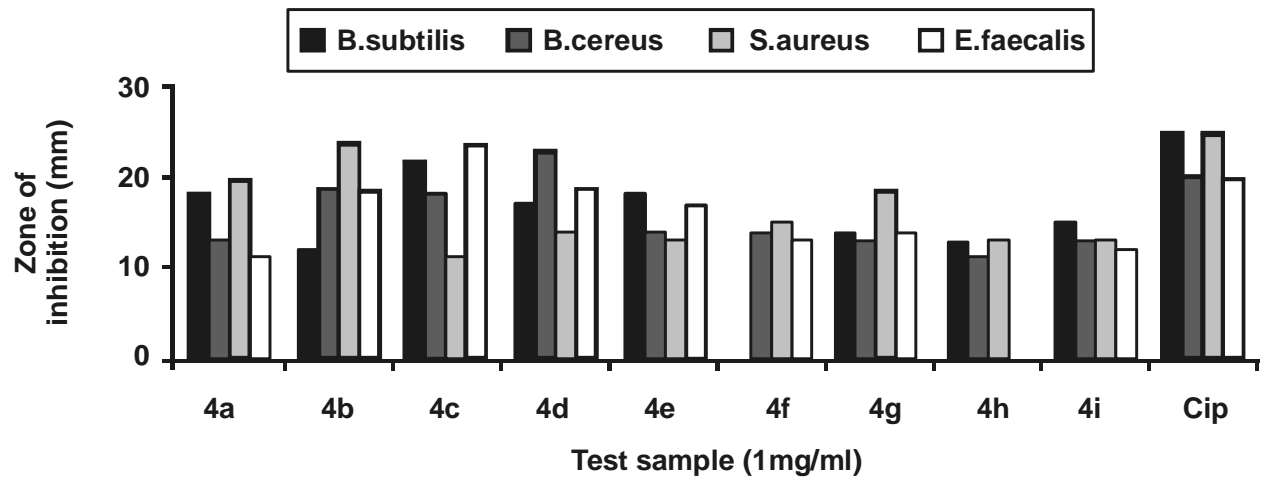

Fig. 1. Antibacterial activity of compds $4 a-i$ against gram poisitive becteria

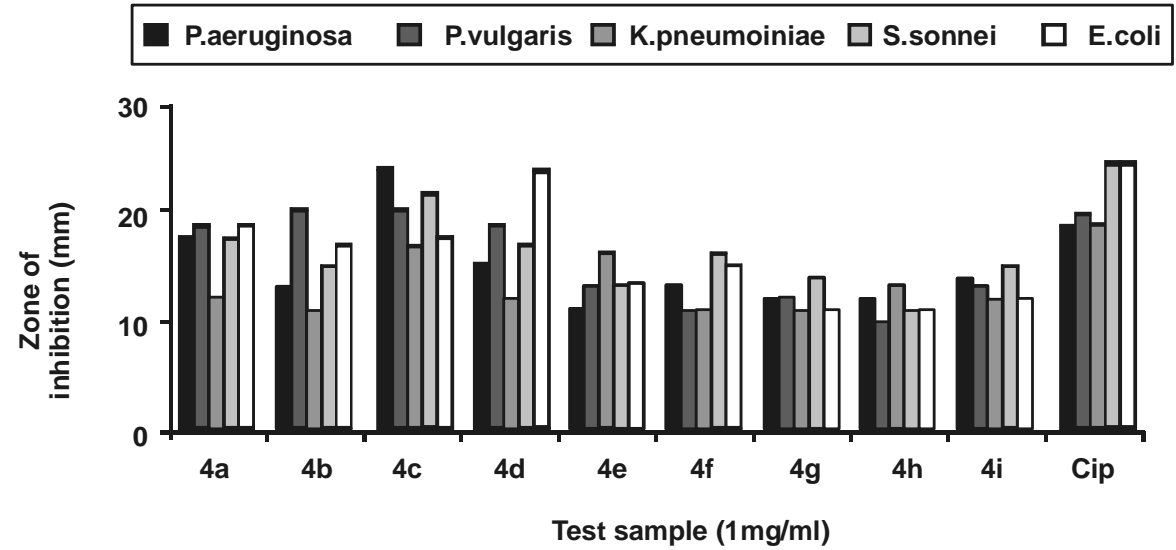

Fig. 2. Antibacterial activity of compds 4 a-i against gram negetive becteria 
B. subtilus . Similarly, compound 4 h was inactive against E. faecalis and $P$. vulgaris. Antibacterial data indicated that compound $\mathbf{4 b}$ showed maximum activity against $P$. vulgaris and exhibited significant activity against $S$.aureus as compared to ciprofloxacin. Compound $\mathbf{4} \mathbf{c}$ was superior in action against $E$. faecalis and $P$. aeruginosa over the standard ciprofloxacin, where as compound 4d showed maximum activity against $B$. cereus and showed significant activity against $E$. faecalis and $E$. coli as compared to standard.

\section{CONCLUSION}

The microbiological results of the present study show a good antibacterial profile in comparison to standard. Although, cyclic amide group (also present in â-lactam ) is required for the antibacterial activity is present in all the tested compounds still, compounds possessing $p$ methyl (4c) or $p$-methoxy moieties (4d) are among the most active compounds in our studies. Moreover, removal of thiomethyl group, in compounds $\mathbf{4 f - 4 i}$, decreases its antibacterial activity against gram positive and gram-negative bacterial strains. Therefore antibacterial screening of thieno pyrimidinones revealed that the compounds having $p$-methyl group (4c)/ $p$ methoxy group (4d) at C-3 and S-methyl group at C-2 positions of C-5, C-6 heterocyclic ring fused pyrimidinones showed significant activity comparable to the standard drug, against grampositive and gram-negative bacterial strains respectively. Hence, a new class of six membered cyclic amide (pyrimidinones), analogues of âlactam have been made available as potential antimicrobial agent.

\section{ACKNOWLEDGEMENT}

We are grateful to Head, Department of Chemistry, Guru Nanak Dev University, Amritsar for providing spectral data for the compounds.

\section{REFERENCES}

Aparna MV, Sati N, Veer VS, Bhosale MS 2005.Synthesis and 5-HT antagonistic activity of some 7- (3substituted amino propoxyl)-4-methyl chromen2ones. Indian J Pharm Sci, 67: 467
Bedi PMS, Mahajan MP, Kapoor VK 2004. Synthesis and evaluation of 1-Aryl-4-N-arylamino-2-phenyl4-thiomethyl-1,3-dienes: A Novel Class of Antibacterial Agents. Indian J Pharm Sci, 66: 112 114.

Cunha BA 1998. Antibiotic Resistance: Control Strategies. Crit Care Clin,14: 309-327.

Dalhoff A 1994. Quinoline Resistance in Pseudomonas areuginosa and Staphylococcus aureus during thearpy and clinical significance. Infection, 22 (Supl. 2): S111-21.

Ehlert K 1999. Methicillin-Resistance in Staphylococcus aureus-Molecular Basis, Novel Targets and Antibiotic Thearpy. Curr Pharm Des, 5(2): 45-55.

Endtz HP, Vanden BN, Verbrugh HA, Van BA 1999. Vancomycin Resistance: Status Quo and Quo Vadis. Eur J Clin Microbiol Infect Dis, 18: 683-690.

File TM 1999. Overview of Resistance in the 1990s. Chest, 115(3): 3S-8 S.

Gootz TD, Brighty K E, Anderson M R, Haskell S L, Sutcliffe J A, Castaldi M J, Miller S A 1992. In Vitro Activity and Synthesis of CP-99219, a Novel 7- (3Azabicyclo[3.1.0]hexyl)-naphthyridone. Paper presented at $32^{\text {nd }}$ Meeting of the ICAAC Anaheim, CA, October 11-14,1992

Hirai K, Hosaka M, Niwata Y, Yasue T, Fukuda H, Ishizaki T, Suzue S, Nishino K 1990. Antibacterial Avtivity of AM-1155, a New Quinoline. Paper presented at $30^{\text {th }}$ Meeting of the ICAAC, Atlanta, GA. October 21-24, 1990.

Hong CY, Kim YK, Chang JH, Kim SH, Choi DH, Kim YZ, Kwak JH 1997. Novel Fluoroquinoline Antibacterial Agents Containing Oxime-Substituted Aminomethyl pyrolidines: Synthesis and Antibacterial Activity of 7-[4- (Aminomethyl)-3(methoxyimino) pyrolidin-1-yl]-1-cyclopropyl-6fluoro-4-oxo-1,4-dihydro[1,8]naphthyridine-3carboxylic Acid (LB20304) . J Med Chem, 40: 35843593.

Ho PL, Yung RH, Tsang DC, Que TL, Ho WH, Seto TK, $\mathrm{Ng}$ WC, Yam and Ng WS 2000. Increasing Resistance of Streptococcus pneumoniae to Fluoroquinones: Results of a Hong Kong Multicentre Study in 2000. J Antimicrob Chemother 48: 659-665.

Maria CP, Claudia M, Michella LR, Federico C, Maurizo B 2004. Solid Phase synthesis of 5,6-disubstituted pyrimidinone and pyrimidindione derivaties. Arkivoc, (v): 349-363.

Petersen U, Bremm KD, Dahoff A, Endermann R, Heilmann W, Krebs A, Schenke T 1996. Synthesis and in Vitro Activity of BAY 12-8039, a new Methoxy Quinoline. Paper presented at the $36^{\text {th }}$ meeting of the ICAAC, New Orleans, LA, September 15-18, 1996.

Witte W 1999. Antibiotic Resistance in Gram-Positive Bacteria Epidemiological Aspects. J Antimicro Chemother, 44A: 1-9.

Weiss K, Restieri C, Gauthier R , Laverdière M , McGeer A et al. 2001. A Nosocomical Outbreak of Fluoroquinoline-Resistant Streptococcus pneumoniae. Clin Infect Dis, 33: 517-522. 